\title{
SMER28 Attenuates Dopaminergic Toxicity Mediated by 6-Hydroxydopamine in the Rats via Modulating Oxidative Burdens and Autophagy-Related Parameters
}

\author{
Shahram Darabi ${ }^{1}$ - Ali Noori-Zadeh ${ }^{2} \cdot$ Farzad Rajaei $^{1} \cdot$ Hojjat Allah Abbaszadeh $^{3} \cdot$ Salar Bakhtiyari $^{4}$. \\ Navid Ahmady Roozbahany ${ }^{5}$
}

Received: 26 April 2018 / Revised: 22 September 2018 / Accepted: 28 September 2018 / Published online: 4 October 2018

(c) Springer Science+Business Media, LLC, part of Springer Nature 2018

\begin{abstract}
Parkinson's disease is the second most common neurodegenerative disease that occurs due to cellular autophagy deficiency and the accumulation of alpha-synuclein in the dopaminergic neurons of the substantia nigra pars compacta ( $\mathrm{SNc}$ ) of the brainstem. The SMER28 (also known as 6-Bromo-N-prop-2-enylquinazolin-4-amine) is an autophagy inducer. In this study, the neuroprotective effects of SMER28 were evaluated on autophagy induction, antioxidant system activation, and microgliosis attenuation. The Parkinson's disease model was developed in the male Wistar rats by injection of 6-OHDA into the left striatum. Apomorphine-induced behavior assessment test and SNc cell counting were performed to investigate the neuroprotective effects of SMER28. This study examined the pharmacological roles of SMER28, especially by focusing on the autophagy (p62/ SQSTM1 and LC3II/LC3I ratio where LC3 is microtubule-associated protein 1A/1B-light chain 3), inhibiting free radicals, and activating the antioxidant system. The levels of malondialdehyde (MDA), reactive oxygen species (ROS), glutathione (GSH), GSH/glutathione peroxidase $\left(\mathrm{GP}_{\mathrm{X}}\right)$, superoxide dismutase (SOD) activity and nuclear factor-erythroid 2-related factor-2 (Nrf2) were measured to evaluate the antioxidant activity of SMER28. Moreover, Iba-1 (ionized calcium binding adaptor molecule, indicating microgliosis) and tyrosine hydroxylase immunoreactivities were evaluated in the SNc. In the behavioral assessment, SMER28 $(50 \mu \mathrm{g} / \mathrm{kg})$ attenuated damages to the SNc dopaminergic neurons, characterized by improved motor function. The tissue observations revealed that SMER28 prevented the destruction of SNc neurons and attenuated microgliosis as well. It also reduced MDA and ROS production and increased GSH, GP ${ }_{\mathrm{X}}, \mathrm{SOD}$, and Nrf2 activities by inducing autophagy (decreasing p62 and increasing LC3II/LC3I ratio). Consequently, possibly with further studies, it can be considered as a drug for neurodegenerative diseases with proteinopathy etiology.
\end{abstract}

Keywords Dopaminergic neurons $\cdot$ Nrf2 $\cdot$ Parkinson's disease $\cdot$ SMER28 $\cdot$ SOD

Shahram Darabi

shahram2005d@yahoo.com

1 Cellular and Molecular Research Center, Qazvin University of Medical Sciences, P.O. BOX: 59811-34197, Qazvin, Iran

2 Department of Clinical Biochemistry, Faculty of Paramedicine, Ilam University of Medical Sciences, Ilam, Iran

3 Hearing Disorders Research Center, Loghman Hakim Hospital, Shahid Beheshti University of Medical Sciences, Tehran, Iran

4 Department of Clinical Biochemistry, Faculty of Medicine, Ilam University of Medical Sciences, Ilam, Iran

5 Raymond Chang School, Ryerson University, Toronto, Canada

\section{Introduction}

Parkinson's disease is a neurodegenerative and movement disorder that affects $50 \%$ of people over the age of 85 years [1]. Even though the etiology of Parkinson's disease is not completely known, a large body of evidence has shown that factors including oxidative stress, inflammation, mitochondrial damage and protein aggregations, such as alpha-synuclein, due to autophagy and antioxidant system dysfunction are involved in the destruction of substantia nigra pars compacta $(\mathrm{SNc})$ dopaminergic neurons.

The term 'autophagy', derived from the Greek meaning 'eating of self'. It is a self-degradative action that is essential for balancing energy sources at critical times in response to nutrient stress. It also plays a housekeeping role in clearing 